\title{
Educação Física Inclusiva: Atitudes dos Docentes
}

\author{
Celina Luísa Raimundo Martins*
}

\begin{abstract}
Resumo: A Educação Física (EF) é vista como um componente curricular privilegiado na Inclusão de estudantes com Necessidades Educativas Especiais (NEE). Pretendeuse com este estudo descrever as atitudes inclusivas de 53 docentes de EF a lecionar em escolas públicas do Distrito do Porto - Portugal. Aplicou-se uma metodologia mista, com recurso a uma escala de atitude e a um grupo de discussão. Os resultados revelaram atitudes positivas, que dependeram de diferentes fatores. Concluiu-se a necessidade de investir na formação específica dos docentes, no apoio multidisciplinar, na diferenciação curricular, nas infraestruturas e na participação ativa de toda a comunidade educativa.
\end{abstract}

Palavras chave: Educação Física. Atitudes. Escola. Inclusão.

\section{INTRODUÇÃo}

No paradigma da escola para todos, também o modo como o desporto é trabalhado na escola regular deve ser contextualizado e reinventado, de modo a garantir a igualdade de oportunidades de participação a todos os estudantes.

A prática desportiva escolar tem sido considerada como um importante facilitador de atitudes inclusivas ao promover o contato e relacionamento social entre os estudantes (BLOCK; RIZZO, 1995, RODRIGUES, 2008, SOUZA; BOATO, 2010, TRIPP; RIZZO; WEBBERT, 2007). Deste modo, o componente curricular de EF deve ser reconhecida como um campo fértil para criatividade e manifestações de valores e sentimentos solidários, contribuindo para que nos espaços escolares se efetive o combate ao preconceito e à

\footnotetext{
*Universidade de Vigo, Vigo, Espanha. E-mail: celinammm@gmail.com
} 
exclusão social (FERNANDES; MÜLLER, 2008). Torna-se assim importante encorajar os docentes a explorarem formas de facilitar a participação efetiva de todos os estudantes (AINSCOW, 1997).

Historicamente, o conceito de escola inclusiva remonta há pelo menos seis décadas, desde a publicação da Declaração Universal dos Direitos Humanos (1948). A sua origem baseou-se num passado altamente influenciado pelo poder, pela institucionalização, normalização, punição e separação entre as pessoas. Também o seu percurso tem sido marcado por práticas educativas excludentes e desigualdades sociais no seio da população escolar.

Os grandes princípios enunciados na Declaração de Salamanca (UNESCO, 1994) propõem a educação de todos os estudantes em escolas regulares, que devem combater atitudes discriminatórias, criando comunidades abertas e solidárias. Este conceito de Escola Inclusiva veio pressupor a existência de um ensino de qualidade para todos, independente da sua especificidade, origem cultural ou qualquer outro aspeto que o diferencie (FERNANDES; MÜLLER, 2008).

A partir de 1960, novos conceitos e práticas começaram a ser introduzidos no âmbito das respostas educativas a dar às crianças e jovens com deficiência. Desde a procura de respostas diferenciadas à necessidade de promover o sucesso para todos os estudantes, o caminho da escola inclusiva tem sido difícil e marcado por perspetivas e atitudes polémicas. Em Portugal datam de 1973/74 importantes diplomas legais publicados pelo Ministério da Educação que assumiram, pela primeira vez, a inclusão de estudantes com deficiência (SANCHES; TEODORO, 2006). Contudo, na maioria das escolas, ainda se verifica a falta de compromisso com essa população e muitas das iniciativas nem sempre produzem os resultados esperados (PERRENOUD, 2001).

Como fonte de mudança da sociedade, a educação inclusiva implica processos dinâmicos adequados a cada cultura e contexto, promovendo o direito universal à educação de qualidade para todos (UNESCO, 1994). Atualmente, novas estratégias são

Movimento, Porto Alegre, v. 20, n. 2, p. 637-657, abr.jun. de 2014. 
usadas como: adequações na estrutura e funcionamento das escolas, a colaboração de técnicos especializados, a diferenciação pedagógica, a individualização dos percursos de formação adequados às necessidades dos estudantes com Necessidades Educativas Especiais (NEE), os estudos dirigidos, entre outras. Em muitos países começaram também a ser integrados no currículo de formação inicial de professores e educadores componentes curriculares respeitantes às NEE com o objetivo de prepará-los para lecionar em classes inclusivas (RODRIGUES, 2006).

Nos ambientes ditos inclusivos deve prevalecer uma cultura inclusiva implicando uma mudança de atitudes no seio da comunidade educativa. Torna-se assim necessário investir na formação humana dos professores e alunos e nas relações famíliaescola, predominando a cooperação entre todos os envolvidos (SANCHES; TEODORO, 2006). O Ensino Cooperativo é portanto, um fator de sucesso essencial, não só porque os professores precisam de suporte, como também adquirirem competências para serem capazes de cooperar com as famílias e profissionais dentro e fora da escola (SANCHES; TEODORO, 2006, AINSCOW, 1997). Neste sentido, só a coesão do coletivo da escola pode incentivar atitudes positivas para se desenvolverem projetos inovadores. Esta questão da partilha deve ser entendida como cultura e não como método ou técnica, assumindo assim uma importância fundamental.

No que respeita à prática desportiva nas escolas regulares, a inclusão ainda não veio renovar, o suficiente, o conteúdo da EF. Este componente curricular continua a englobar essencialmente modalidades coletivas e competitivas, mantendo uma forte ênfase sobre o desempenho e a excelência. Estas caraterísticas tendem a reduzir a participação dos estudantes com NEE em igualdade de oportunidades com os seus pares (HAYCOCK; SMITH, 2011a).

Se tomarmos em consideração a Carta Europeia do Desporto para Todos, que estipula que "todo o indivíduo tem direito à prática desportiva" (CONSELHO DA EUROPA, 1988, p. 8), torna-se evidente que o desporto deve ser planejado para todos,

Movimento, Porto Alegre, v. 20, n. 2, p. 637-657, abr.jun. de 2014. 
independentemente da sua condição individual. Embora diferente e com limitações, uma pessoa com deficiência possui legislação própria que a protege e lhe assegura o direito à prática desportiva nos variados domínios sociais assim como no âmbito escolar.

A escola inclusiva repousa sobre a capacidade de iniciativa e os valores do professor (RODRIGUES, 2006). Contudo, no mundo da prática desportiva em meio escolar, os docentes não têm conseguido desenvolver um ensino articulado com a nova realidade social heterogênea (NASCIMENTO et al, 2009). É, portanto incontestável que a área da EF especial ou adaptada carece de melhor preparação e interesse por parte dos profissionais. Recentemente, esta formação específica começou a ser incluída nos programas curriculares das universidades (MORLEY et al., 2005; RODRIGUES, 2006).

A experiência e os conhecimentos sobre as NEE são considerados fatores da maior importância para o desenvolvimento de atitudes positivas, uma vez que lecionar em classes inclusivas requer um maior dinamismo e criatividade por parte dos docentes (JEONG; BLOCK, 2011). Torna-se também importante fazer a ligação entre o conteúdo da $\mathrm{EF}$, os estudantes e o docente, uma vez que a prática desportiva se revela um instrumento privilegiado de intervenção com estudantes portadores de deficiência.

No universo desportivo existem várias vertentes aplicáveis às pessoas com deficiência e promotoras de integração social. Compete ao docente de EF intervir na formação de valores inclusivos, evitando preconceito relacionado com qualquer tipo de diferença (RODRIGUES; DARIDO, 2011). Deve assim fomentarse a solidariedade e a cooperação entre os estudantes, eliminando as variadas formas de exclusão. Ross (2004) salienta ainda que a inclusão dos estudantes com NEE deve ser da responsabilidade de toda a comunidade escolar, que deve sentir-se comprometida na plena inclusão destes estudantes.

Para além do conceito da diferença e das práticas inclusivas, devemos reconhecer a árdua tarefa dos docentes que lecionam em classes heterogêneas. Pois não basta que a escola assuma o

Movimento, Porto Alegre, v. 20, n. 2, p. 637-657, abr.jun. de 2014. 
discurso da diferença, é preciso colocar a própria diferença em discussão. São vários os autores que reconhecem que as atitudes inclusivas dos docentes face à inclusão escolar são uma condição de extrema importância para que este processo se efetive com qualidade (LAMBE; BONES, 2006, DUNN, 2008, GALVÃO, 2002, JEONG; BLOCK, 2011, JERLINDER, DANERMARK; GILL, 2010, SOUZA; BOATO, 2010).

Não há dúvida de que o docente exerce uma função única dentro da escola. Ele é o elemento de ligação entre a escola, a sociedade, o conhecimento e o aluno (DUNN, 2008, GALVÃO, 2002). A sua intervenção na inclusão escolar é uma condição de extrema importância para se alcançar a igualdade de oportunidades entre todos os estudantes. Um profissional de ensino, capacitado e interessado, pode desenvolver estratégias bem planejadas, ao avaliar constantemente e ao mudar as atitudes discriminatórias (FREITAS, 2008). Espera-se que os docentes tenham uma atitude acolhedora da diversidade e estejam disponíveis para aplicar pedagogias inovadoras em sala de aula. Há, portanto necessidade de adotar uma postura crítica e novos programas que atendam a diversidade (DOMÍNGUEZ ALONSO; PINO 2008).

"Os recursos são secundários. O importante é a atitude da escola e do docente" (RODRIGUES, 2006, p. 7). De acordo com o autor, perante o idealismo que associa a inclusão aos direitos humanos e à justiça social é compreensível que a força fundamental da promoção de um determinado programa repouse nas atitudes, na vontade e na ética dos docentes. Outros estudos (JEONG; BLOCK, 2011, QI; HA, 2012) confirmam que as crenças e atitudes dos docentes de EF face à inclusão de estudantes com NEE influenciam a sua pedagogia, reforçando a teoria do comportamento planejado. Efetivamente, se valorizarmos as atitudes, outros fatores, como por exemplo, os recursos podem ser menos valorizados (AVRAMIDIS; NORWICH, 2002).

Considerando estas constatações, chegou-se às questões geradoras do problema de estudo que se consolidou pela colocação de algumas questões, que se enunciam: Que Atitudes apresentam os

Movimento, Porto Alegre, v. 20, n. 2, p. 637-657, abr.jun. de 2014. 
docentes de EF sobre a Inclusão de Estudantes com Necessidades Educativas Especiais? Quais são os fatores que mais influenciam as suas atitudes inclusivas? Que barreiras existem ao desenvolvimento da EF inclusiva? Quais são os fatores facilitadores?

Face às questões colocadas, o principal objetivo foi descrever as atitudes da comunidade Educativa sobre EF Inclusiva, pelo que se formularam as hipóteses do estudo relacionadas com a natureza das atitudes, concretamente no que respeita à inferência das caraterísticas pessoais e profissionais dos participantes do estudo.

\section{Método}

Para responder ao problema de estudo partiu-se do método dedutivo e optou-se por um estudo de enfoque interpretativo e desenho não experimental, através de uma investigação empírica e de campo. A perspetiva metodológica da pesquisa combinou uma abordagem mista, de natureza quantitativa e qualitativa, para a aproximação à realidade estudada.

A amostra foi constituída por 53 docentes de EF, que lecionavam em escolas do Ensino Básico () do distrito do Porto no ano letivo 2011/2012, sendo $49,1 \%$ do gênero masculino e 50,9\% do feminino. 18,9\% tinham menos que 30 anos; 52,8\% situavam-se entre 30 e 40 anos; $17 \%$ entre 41 e 50 anos e 11,3\% apresentaram mais de 50 anos.

Quanto ao grau academico, 77,4\% tinham licenciatura e $22,6 \%$ mestrado. Relativamente à experiência profissional, os

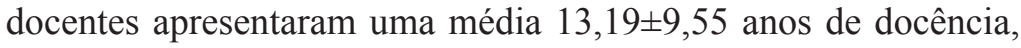
sendo que $64,2 \%$ possuía experiência em classes inclusivas.

Em relação ao nível de ensino, 41,5\% lecionavam no $3^{\circ}$ Ciclo e Secundário, $34 \%$ no $1^{\circ}$ Ciclo e $24,5 \%$ eram docentes do $2^{\circ}$ Ciclo do Ensino Básico. Quanto à formação específica, 73,6\% dos docentes tiveram na sua formação inicial componentes curriculares sobre NEE e 47,2\% participaram em Ações de Formação sobre Escola Inclusiva.

Movimento, Porto Alegre, v. 20, n. 2, p. 637-657, abr./jun. de 2014. 
Numa segunda fase do estudo, formou-se um grupo de discussão, composto de 9 participantes: 5 docentes de EF (3 do gênero masculino e 2 do feminino), com uma média de idade de 39,4 anos; 2 Tutores do gênero feminino com 39 e 42 anos de idade e 2 alunas do $2^{\circ}$ e $3^{\circ}$ Ciclo do Ensino Básico, que estudaram em classes inclusivas com 11 e 14 anos de idade respetivamente.

Como foi realizada uma investigação em meio escolar, este projeto foi submetido à aprovação pela Direcção-Geral de Inovação e de Desenvolvimento Curricular do Ministério da Educação de Portugal, cumprindo o estipulado no Despacho n. ${ }^{0} 15847 / 2007$ publicado no DR $2^{\text {a }}$ série n. ${ }^{\circ} 140$ de 23 de Julho.

Após obter também a aprovação das direções das 17 escolas alvo, aplicou-se o questionário desenvolvido para avaliar a Atitude Global Inclusiva: "A EF e a Inclusão de alunos com NEE". A primeira parte do questionário reportou-se às características Pessoais e Profissionais dos participantes. Na segunda parte, os docentes indicaram o seu grau de concordância com 12 afirmações sobre o tema, segundo uma escala de Likert (Visual Analogue Scales), de 1 a 5. Em seguida, ordenaram por ordem de prioridade as condições para o sucesso da EF Inclusiva e as dificuldades pedagógicas em classes inclusivas. A escala aplicada revelou-se um instrumento de medida válido e confiável com boa consistência interna (Alpha de Cronbach=0,71).

Os dados quantitativos foram analisados no programa SPSS (Statistical Package for the Social Sciences) versão 19.0 para o Windows. Recorreu-se à estatística descritiva e inferencial, através dos testes $t$ de Student, One Way Anova e Correlação de Pearson, considerando a independência dos grupos.

A técnica do grupo de discussão foi usada após a pesquisa quantitativa, com o intuito de integrar diferentes perspetivas e de esclarecer e interpretar melhor os resultados. Organizou-se um grupo de discussão online (ef-inclusiva), em que se aplicou uma matriz guia de coleta de dados procurando descrever melhor a realidade da inclusão em EF e identificar perspetivas de melhoria. Realizou-se uma análise de conteúdo sistematizada da informação resultante.

Movimento, Porto Alegre, v. 20, n. 2, p. 637-657, abr.jun. de 2014. 


\section{Resultados}

A média de Atitude Global Inclusiva dos docentes de EF apresentou um valor de $\mathrm{M}=3,29 \pm 0,34$ na escala de 1 a 5 . O valor mínimo obtido foi de $\mathrm{M}=2,42$ e o máximo de $\mathrm{M}=4,08$.

O item mais valorizado pelos participantes foi o da importância da formação especializada dos docentes de EF para poder dar uma resposta adequada a todos os estudantes $(\mathrm{M}=4,08 \pm 1,11)$.

Face às dificuldades deste processo, os docentes parecem concordar com uma redução da sua eficácia pedagógica em classes inclusivas $(\mathrm{M}=4,06 \pm 1,06)$ e que a inclusão obriga a alterar as atividades normais das aulas $(\mathrm{M}=4,06 \pm 0,91)$.

No grupo de discussão, os docentes explicaram estas dificuldades porque "cada aluno com NEE necessita de um currículo adaptado à sua dificuldade" (docente 3 ), a estudante 2 argumentou que " há estudantes que não conseguem fazer todos exercícios; também não têm os materiais que precisam" "a exigência na planificação deriva da ética profissional do Docente de EF... há sempre forma de planificar uma atividade para um aluno especial se o docente conhecer bem esse aluno e os seus limites e capacidades" (docente 1). Revelaram também que "o currículo é pouco flexível, seria necessário uma variedade de currículos para os diferentes estudantes com NEE" (docente 5).

Face aos benefícios da inclusão, os docentes manifestaram concordar $(\mathrm{M}=4,06 \pm 0,90)$ que a presença de um aluno com NEE proporciona novas situações de aprendizagem para todos os estudantes; que atenua as diferenças entre ele e os seus pares $(\mathrm{M}=$ $3,70 \pm 0,93)$ não interferindo com o seu progresso $(\mathrm{M}=3,60 \pm 1,21)$.

Por outro lado, foi evidenciada alguma discordância diante do fato da heterogeneidade das classes não ser fator de insucesso escolar $(\mathrm{M}=2,94 \pm 1,42)$, que os pares que interagem com os estudantes com NEE têm menos possibilidades de se desenvolverem $(M=1,6 \pm 1,01)$ e que a inclusão não é benéfica para o aluno com NEE nem para os pares $(M=2,21 \pm 1,26)$.

Movimento, Porto Alegre, v. 20, n. 2, p. 637-657, abr.jun. de 2014. 
No que respeita às consequências da inclusão para os pares, o tutor 2 referiu que "as crianças que convivem com a diferença tornam-se seres humanos melhores, mais tolerantes e compreensivos, com maior consciência moral e de cidadania. Já as crianças com deficiência têm uma maior interação social $[\ldots]$ ".

Tendo em vista os fatores mais importantes para o sucesso da inclusão em EF, os docentes valorizaram em primeiro lugar a formação específica na área das $\operatorname{NEE}(5,68 \pm 1,59)$ (tabela 1) seguida do apoio de técnicos especializados $(5,25 \pm 1,85)$. Com valores muito aproximados valorizaram as infraestruturas $(3,60 \pm 1,75)$, as metodologias de ensino $(3,64 \pm 1,50)$ e as atitudes dos docentes $(3,66 \pm 1,90)$. Em penúltimo lugar surgiram as atitudes $(3,32 \pm 1,99)$ e em último o processo de avaliação $(2,94 \pm 1,83)$.

Tabela 1: Fatores Necessários à Inclusão

\begin{tabular}{cccccccc}
\hline & Formação & Técnicos & Materiais & Metodologias & Docentes & Atitudes & Avaliação \\
\hline Mean & 5,68 & 5,25 & 3,66 & 3,64 & 3,60 & 3,32 & 2,94 \\
\hline SD & 1,591 & 1,849 & 1,901 & 1,495 & 1,747 & 1,988 & 1,834 \\
\hline
\end{tabular}

Fonte: Elaboração do Autor

Em relação à colaboração entre docentes e técnicos, o grupo de discussão referiu também que "é muito importante a colaboração dos docentes do ensino especial e dos psicólogos para se debaterem em conjunto a melhor forma de integrar os estudantes com NEE" (tutor 2).

Como as principais dificuldades no processo de Inclusão, os docentes realçaram falta de formação $(6,67 \pm 2,86)$, o apoio técnico $(6,89 \pm 2,23)$ e o número de estudantes das classes $(6,92 \pm 3,20)$ (tabela 2).

Tabela 2: Dificuldades na Inclusão em EF

\begin{tabular}{ccccccccccc}
\hline & Apoio & $\begin{array}{c}\text { Forma- } \\
\text { ção }\end{array}$ & $\begin{array}{c}\text { Disponi- } \\
\text { bilidade }\end{array}$ & $\begin{array}{c}\text { Expe- } \\
\text { riencia }\end{array}$ & Métodos & Número & Aluno & Família & $\begin{array}{c}\text { Mate- } \\
\text { riais }\end{array}$ & $\begin{array}{c}\text { Precon- } \\
\text { ceito }\end{array}$ \\
\hline Mean & 6,89 & 6,92 & 4,28 & 5,26 & 5,60 & 6,77 & 5,94 & 5,45 & 5,57 & 2,28 \\
\hline SD & 2,23 & 3,198 & 2,273 & 2,595 & 2,323 & 2,860 & 2,397 & 2,325 & 2,892 & 2,421 \\
\hline
\end{tabular}

Fonte: Elaboração do Autor

Movimento, Porto Alegre, v. 20, n. 2, p. 637-657, abr.jun. de 2014. 
Também o grupo de discussão realçou as dificuldades de formação, de apoio e do número de estudantes nas classes: "falta preparação/formação adequada" (docente 3); "os professores também precisam de apoio (estudante 1); "...falta de técnicos especializados para acompanharem os estudantes nas aulas de EF" (docente 5); ..."sentem muita exigência, porque não têm formação ou muitas das vezes não a procuram. Por outro lado, as turmas são demasiado grandes e a diferenciação torna-se difícil" (tutor 2).

Em $4^{\circ}$ lugar foi classificada a problemática do aluno $(5,94 \pm 2,40)$, seguida das dificuldades decorrentes dos métodos de ensino a se aplicar $(5,60 \pm 2,32)$, da falta de infraestruturas e materiais adaptados $(5,57 \pm 2,89)$ e do apoio da família $(5,45 \pm 2,33)$.

Foram também destacados impedimentos relativos à diferenciação curricular: "o currículo é muito rígido e pouco flexível. Um currículo mais variado, mais adequado às classes com estudantes NEE podia colocar estes estudantes em paridade com os companheiros" (docente 4). Também foi reforçada a ideia de que "a falta de condições físicas adequadas na escola dificulta a inclusão" (docente 3); e que "na maioria das escolas falta material didático adaptado" (docente 5).

As dificuldades menos valorizadas foram: a experiência profissional $(5,26 \pm 2,60)$, a disponibilidade dos docentes $(4,28 \pm 2,27)$ e em último o preconceito $(2,2 \pm 2,42)$.

No estudo inferencial, as variáveis gênero e anos de docência não influenciaram as atitudes dos docentes $(p<0,05)$. Em relação à idade, foi o grupo com menos de 30 anos que demonstrou sentir menos a redução da eficácia pedagógica em classes inclusivas $(\mathrm{M}=3,30 \pm 1,25 ; \mathrm{p}=0,031)$ e o grupo entre 30 a 40 anos foi o que mais concordou com esta afirmação $(M=4,39 \pm 0,83)$.

Também, no que respeita ao grau academico, os docentes com grau de mestre concordaram mais $(M=4,67 \pm 0,49 ; \mathrm{p}=0,041)$ que a sua eficácia diminui nas classes com estudantes com NEE do que o grupo de docentes licenciados $(\mathrm{M}=3,85 \pm 1,12)$.

Movimento, Porto Alegre, v. 20, n. 2, p. 637-657, abr.jun. de 2014. 
Comparativamente ao Ciclo de Ensino, os resultados indicaram que as atitudes inclusivas dos docentes diminuíram à medida que aumentava o ciclo de ensino (tabela 3 ).

Tabela 3: Atitude em função do Ciclo de Ensino

\begin{tabular}{|c|c|c|c|c|c|}
\hline Ciclo de Ensino & $\mathbf{N}$ & Mean & Std. Deviation & F & Sig. \\
\hline $1^{\circ}$ Ciclo & 18 & 3,3750 & ,36408 & \multirow{3}{*}{2,529} & \multirow{3}{*}{,090 } \\
\hline $2^{\circ}$ Ciclo & 13 & 3,3654 & ,30908 & & \\
\hline $3^{\circ}$ Ciclo & 22 & 3,1667 & ,30211 & & \\
\hline
\end{tabular}

Fonte: Elaboração do Autor

Os resultados apontaram para a importância dos cursos de formação básica dos docentes incidirem na temática da inclusão. Os resultados indicaram que o grupo sem formação sente mais $(\mathrm{p}=0,017)$ a necessidade de alterar as atividades normais das aulas $(M=4,50 \pm 0,65)$ do que o grupo que recebeu formação $(\mathrm{M}=3,69 \pm 1,15)$. Este grupo sem formação também deu mais importância à Formação Específica na área das NEE como uma condição necessária ao sucesso da Inclusão $(M=5,36 \pm 1,69)$ do que o grupo com formação $(M=6,57 \pm 0,76 ; p=0,013)$.

Conjuntamente, a frequência de ações de formação específica inferiu de forma significativa nas atitudes. O grupo que não realizou ações pareceu perceber mais a redução da sua eficácia pedagógica e necessitar mais de formação (tabela 4).

Tabela 4: Atitude em função da Frequências de Ações de Formação Específica

\begin{tabular}{|c|c|c|c|c|c|c|c|c|c|}
\hline & $\begin{array}{c}\text { Ações de } \\
\text { Formação }\end{array}$ & $\mathbf{N}$ & Média & D P & F & Sig. & $T$ & Df & $\begin{array}{l}\text { Sig. } \\
(2-t)\end{array}$ \\
\hline $\begin{array}{c}\text { Redução } \\
\text { da Eficácia } \\
\text { Pedagógica }\end{array}$ & $\begin{array}{l}\text { Sim } \\
\text { Não }\end{array}$ & $\begin{array}{l}25 \\
28\end{array}$ & $\begin{array}{l}3,68 \\
4,39\end{array}$ & $\begin{array}{c}1,145 \\
, 875\end{array}$ & 2,488 & ,121 & $\begin{array}{l}2,563 \\
2,524\end{array}$ & $\begin{array}{c}51 \\
44,755\end{array}$ & $\begin{array}{l}, 013 \\
, 015\end{array}$ \\
\hline $\begin{array}{c}\text { Necessidade } \\
\text { de } \\
\text { Formação }\end{array}$ & $\begin{array}{l}\text { Sim } \\
\text { Não }\end{array}$ & $\begin{array}{l}25 \\
28\end{array}$ & $\begin{array}{l}3,68 \\
4,43\end{array}$ & $\begin{array}{c}1,282 \\
, 790\end{array}$ & 7,767 & ,007 & $\begin{array}{l}2,589 \\
2,523\end{array}$ & $\begin{array}{c}51 \\
39,050\end{array}$ & $\begin{array}{l}, 013 \\
, 016\end{array}$ \\
\hline
\end{tabular}

Fonte: Elaboração do Autor

Movimento, Porto Alegre, v. 20, n. 2, p. 637-657, abr.jun. de 2014. 
A inferência da variável experiência em inclusão demonstrou que o grupo sem experiência necessita mais de formação especializada para dar resposta adequada aos estudantes com NEE, já o grupo com experiência percebeu mais dificuldades pela falta de infraestruturas e materiais adequados (tabela 5).

Tabela 5: Atitude em função da Experiência em Inclusão

\begin{tabular}{|c|c|c|c|c|c|c|c|c|c|}
\hline & Experiência & $\mathrm{N}$ & M & SD & F & Sig. & $\mathrm{T}$ & $\mathrm{df}$ & $\begin{array}{l}\text { Sig. } \\
(2-t)\end{array}$ \\
\hline \multirow{2}{*}{$\begin{array}{l}\text { Valorização } \\
\text { da Formação }\end{array}$} & Sim & 34 & 3,85 & 1,234 & \multirow[t]{2}{*}{4,463} & \multirow[t]{2}{*}{,040 } & 2,015 & 51 & 049, \\
\hline & Não & 19 & 4,47 & ,697 & & & 2,340 & 50,9 & 023 \\
\hline \multirow{2}{*}{$\begin{array}{l}\text { Dificuldades } \\
\text { por falta de } \\
\text { Materiais }\end{array}$} & Sim & 34 & 6,18 & 2,790 & \multirow[t]{2}{*}{,255 } & \multirow[t]{2}{*}{,616 } & 2,124 & 51 & 039 \\
\hline & Não & 19 & 4,47 & 2,816 & & & 2,118 & 37,08 & ,041 \\
\hline
\end{tabular}

Fonte: Elaboração do Autor

\section{Dıscussão}

Os resultados sugeriram que as atitudes dos docentes de EF foram, na maioria das vezes, favoráveis à inclusão de estudantes com NEE nas suas aulas. As atitudes pareceram depender de vários fatores como a idade, o nível de ensino, a formação específica em NEE e a experiência em classes inclusivas.

Também outros autores chegaram a resultados similares e concluíram que os docentes estão disponíveis para a inclusão (HODGE et al., 2004, JERLINDE; DANERMARK; GILL, 2010), embora não se considerem devidamente capacitados para lecionar aulas inclusivas (SOUZA; BOATO, 2010).

Tal como no presente estudo, também Hodge et al. (2004) encontrou como maiores dificuldades a falta de preparação dos docentes e poucos recursos humanos e materiais das escolas. Outros como Morley et al. (2005) confirmam a importância do apoio de técnicos especializados para se explorarem as possibilidades de participação no componente curricular de EF.

Movimento, Porto Alegre, v. 20, n. 2, p. 637-657, abr./jun. de 2014. 
A formação em NEE e/ou atividade física adaptada revelouse uma variável determinante nas atitudes inclusivas dos docentes, sendo que a grande maioria evidenciou a necessidade de mais formação específica. Do mesmo modo, nos estudos de outros autores (BLOCK; RIZZO, 1995, HUTZLER, 2003, KOWALSKI; RIZZO, 1996, MONTEIRO, 2008, RIZZO; VISPOEL, 1991, RIZZO; KIRKENDALL, 1995), constatou-se que os docentes sem formação apresentavam atitudes negativas. Os futuros docentes têm de ser formados para conhecer e aplicar os conteúdos deste componente curricular de forma diferenciada (RODRIGUES, 2008, SHARMA; FORLIN; LOREMAN, 2008). Também, Nascimento et al. (2009), concluíram que a formação contínua é mais relevante para a atuação do profissional nas aulas de EF no contexto inclusivo que a formação inicial. Contudo, Pinto Pires e Condado, (2012), ao analisarem o currículo dos cursos de EF das universidades portuguesas, concluíram que não existe background na formação dos docentes para que os conceitos de inclusão se concretizem. Efetivamente a formação sobre EF inclusiva não deve ser restrita ao curso de base. Os profissionais devem dotar o seu currículo de conhecimentos e experiências ao longo do percurso profissional (BLOCK; RIZZO, 1995, PARRILLA; MORIÑA, 2006, RIZZO; KIRKENDALL, 1995).

Assim como Hutzler (2003), comprovou-se que a experiência em ensino inclusivo, contribuiu significativamente para atitudes favoráveis. Rodrigues $(2006$, p. 307) chama a atenção para que o processo de formação específica seja ligado à prática profissional, para que os docentes possam contar com uma equipe de apoio.

Em consonância com outros estudos, não se verificou a influência da variável gênero nas atitudes inclusivas dos docentes (KOWALSKI; RIZZO, 1996, MONTEIRO, 2008; RIZZO; DAVIS; TOUSSAINT, 1994, TRIPP; RIZZO; WEBBERT, 2007). Contudo, existem outros que evidenciaram uma atitude mais inclusiva para o gênero feminino (FOLSOM-MEEK; et al, 1999, GORGATTI, 2009, HUTZLER, 2003).

Movimento, Porto Alegre, v. 20, n. 2, p. 637-657, abr.jun. de 2014. 
O grau acadêmico também não se relacionou com a formação na área das NEE nem influenciou a eficácia pedagógica. Do mesmo modo, outros autores (CORTEZ, 2008, HAYCOCK; SMITH, 2011, KOWALSKI; RIZZO, 1996), demostraram que o grau de competência percebida é o melhor preditor de atitudes favoráveis e não o grau de formação acadêmica.

Os docentes de EF de níveis de ensino inferiores demonstraram atitudes mais positivas. São vários os autores que preconizam esta concepção, dado que a inclusão é vista de forma mais positiva em relação aos estudantes mais novos (DEPAUW; DOLL-TEPPER, 2000, KOWALSKI; RIZZO, 1996, RIZZO; VISPOEL, 1991). Neste sentido, também Domínguez Alonso e Pino (2009) indicam que medidas de atenção à diversidade dependem do nível e ensino e do tipo de necessidades de apoio educativo que os estudantes apresentam. Destaca-se a constribuição de Salvia e Munson (1986), ao concluírem que à medida que estas crianças crescem e evoluem na hierarquia escolar, os docentes tendem a centrar-se mais na matéria transmitida, relegando para segundo plano as diferenças e dificuldades da classe.

Autores como Block e Rizzo (1995); Rizzo e Vispoel (1991) e Rodrigues (2005), confirmam, como neste estudo, que docentes com experiência inclusiva ultrapassam mais facilmente os preconceitos e apresentam atitudes mais favoráveis. Neste sentido, Haycock e Smith (2011), indicam que os profissionais de educação só podem perceber a inclusão de forma adequada estando dentro do contexto. Também Ainscow (1997) e Karagiannis, Stainback e Stainback (1999), admitem que a escola inclusiva incita o dinamismo dos seus profissionais, sendo que a conjuntura da inclusão leva as escolas e os seus profissionais a refletirem mais na tentativa de conseguir novas formas de trabalhar.

A estreita colaboração entre profissionais, famílias e comunidade é indicada como um fator de sucesso (MANTOAN, 2003, SANCHES, 2006, AINSCOW, 1997, HAYCOCK; SMITH, 2011). Também, o currículo do componente curricular foi apontado

Movimento, Porto Alegre, v. 20, n. 2, p. 637-657, abr.jun. de 2014. 
como pouco flexível, dificultando a diferenciação pedagógica e a adoção de metodologias adequadas a todos os estudantes, pelo que se destacam as competências dos docentes ao nível da gestão flexível do currículo e dos processos de diferenciação pedagógica e de aplicar metodologias de trabalho em equipe. Neste sentido, alguns autores (BIEGER, 2012, CHICON, 2008, HOWES; GRIMES; SHOHEL, 2011, KHOCHEN; RADFORD, 2012, SHARMA; FORLIN; LOREMAN, 2008) apontam para a importância dos docentes de EF desenvolverem no seu percurso formativo, uma pedagogia crítica e flexível, em colaboração com toda a comunidade educativa. Mas para que este objetivo se torne possível, é realçada a construção de uma cultura inclusiva no seio da comunidade educativa, por meio de uma relação de diálogo mútuo, estabelecido na compreensão dos diversos pontos de vista, onde cada parte envolvida possa dar a sua contribuição, mas sempre em articulação e com metas comuns. Outros estudos também confirmam que a melhoria das práticas inclusivas implica a valorização da participação das famílias na escola. Esta participação leva a um conhecimento mútuo sobre a educação dos alunos e aumenta a confiança e a cooperação, o que traz repercussões muito positivas no processo de ensino aprendizagem (DOMÍNGUEZ ALONSO; PINO, 2009, RODRIGUES, 2008). Efetivamente, o processo de qualificação e capacitação dos professores, em um trabalho conjunto com a comunidade escolar e os pais dos alunos, pode ser o melhor caminho facilitador da inclusão (RODRIGUES, 2008).

\section{Conclusões}

Desta pesquisa concluiu-se que o êxito da educação futura dependerá de um trabalho conjunto e colaborativo.

O apoio do governo é indispensável no que se refere à oferta formativa especializada e à melhoria dos recursos materiais e apoio técnico das escolas. Contudo, é na capacitação dos vários agentes educativos que deve assentar a transformação do próprio sistema de ensino. Para que a inclusão se efetive nos diferentes contextos sociais,

Movimento, Porto Alegre, v. 20, n. 2, p. 637-657, abr.jun. de 2014. 
é portanto necessário promover iniciativas de formação e reflexão intersetoriais, entre profissionais da escola, da saúde e dos serviços sociais, ampliando os vínculos com as famílias e comunidade.

Realça-se a necessidade da formação de professores para o desenvolvimento de uma pedagogia mais inclusiva que contemple a flexibilidade curricular e metodologias de ensino diversificadas. Nota-se ainda a necessidade da restruturação do currículo do componente curricular de EF adotando um caráter menos competitivo, mais flexível e solidário. Mas só uma estreita cooperação entre todos os envolvidos pode fomentar na escola uma cultura cada vez mais inclusiva, possibilitando o desenvolvimento integral de todos os alunos.

\section{Inclusive Physical Education: Teachers' Attitudes}

Abstract: The Physical Education (PE) is perceived as a prime area for Inclusion of Students with Special Educational Needs (SEN). The intention of this study was to describe the inclusive attitudes of $53 \mathrm{PE}$ teachers from Portuguese public schools. It was applied a mixed methodology, using a scale of attitude and a discussion group. The results revealed positive attitudes, that depended on different factors. It was concluded a need to invest in specific formation of teachers, in the multidisciplinary support, in curricular differentiation, on infrastructures and in the active participation of the whole educational community.

Keywords: Physical Education. Attitudes. School. Inclusion.

\section{Educación Física Inclusiva: Actitudes del Profesorado \\ Resumen: La Educación Física se percibe como una materia privilegiada para la Inclusión de Estudiantes con Necesidades Educativas Especiales. El objetivo de este estudio fue describir las actitudes inclusivas de 53 docentes de Educación Física de escuelas públicas portuguesas. Se aplicó una metodología mixta, usando una escala de actitud y un grupo de discusión. Los resultados revelaron actitudes positivas, que se relacionan con distintos factores. Se concluye que es preciso invertir en la formación específica de los profesores, en el apoyo multidisciplinar, en la diferenciación curricular, en infraestructuras y en la participación activa de toda la comunidad educativa. \\ Palabras clave: Educación Física. Actitudes. Escuela. Inclusión.}




\section{REFERÊNCIAS}

AINSCOW, Mel. Educação para todos: torná-la uma realidade. In: CAMINHOS para as escolas inclusivas. Lisboa: Instituto de Inovação Educacional, 1997. p. 11-31.

AINSCOW, Mel. Exploring links between special needs and school improvement. Support for Learning - British Journal of Special Education, Cumbria, v. 13, n. 2, p. 70-75, maio 1998.

AVRAMIDIS, Elias; NORWICH, Brahm. Teachers' attitudes towards integration/ inclusion: a review of the literature. European Journal of Special Needs Education, Coventry, v. 17, n. 2, p. 129-147. 2002.

BAUMAN, Zygmunt. Life in fragments: essays in postmodern morality. Oxford: Blackwell, 1995.

BLOCK, Martin; RIZZO, Terry. Attitudes and Attributes of Physical Educators Associated with Teaching Individuals with Severe and Profound Disabilities. Journal of the Association for Persons with Severe Handicaps, Seattle, v. 20, n. 1, p. 80-87. Sept.1995.

CHICON, José Francisco. Inclusão e exclusão no contexto da educação física escolar. Movimento, Porto Alegre, v. 14, n. 1, p. 13-38, jan./abr. 2008.

CONSELHO DA EUROPA. Carta europeia do desporto para todos: as pessoas deficientes. Desporto e Sociedade, Lisboa, n. 105, 1988.

DEPAUW, Karen; DOLL-TEPPER, Gudrun. Toward progressive inclusion and acceptance: Myth or reality? The inclusion debate and bandwagon discourse. Adapted Physical Activity Quarterly, Champaign, v. 17, n. 2, p. 135-143, Apr. 2000.

DOMÍNGUEZ ALONSO, José; PINO, Margarita Rosa. Las conductas problemáticas en el aula: propuesta de actuación. Revista Complutense de Educación, Madrid, v. 19, n. 2, p. 447-457, abril. 2008.

DOMÍNGUEZ ALONSO, José; PINO, Margarita Rosa. Evaluación de las Medidas de Atención a la Diversidad en la Educación Primaria en Galicia: Impacto escolar. Revista Española de Orientación y Psicopedagogía, Madrid, v. 20, n. 2, p.123134, 2. cuatrimestre. 2009.

DUNN, John. Special physical education. Adapted Physical Activity Quarterly, Champaign, v. 25, n. 2, p. 174-176, 2 Apr. 2008.

ELHOWERIS, Hala; ALSHEIKH, Negmeldin. Teachers' attitudes toward inclusion. International Journal of Special Education, Manchester, v. 21, n. 1, p. 115-118. 2006.

FERNANDES, Luciane Pereira; MÜLLER, Verônica Regina. Exclusão e Inclusão Social: contribuições e experiências Inclusivas na educação física. Curitiba: SEED/PR, 2009. Disponível em: <http://www.diaadiaeducacao.pr.gov.br/portals/ pde/ arquivos/949-4.pdf> Acesso em: 19 abr. 2013.

Movimento, Porto Alegre, v. 20, n. 2, p. 637-657, abr.jun. de 2014. 
FOLSOM-MEEK, Sherry et al. Effects of academic major, gender, and hands-on experience on attitudes of preservice professionals. Adapted Physical Activity Quarterly, Champaign, v. 16, n. 4, p. 389-402, Oct.1999.

FREITAS, Neli Klix. Social and educational inclusion: the process and the students' evaluation. Ensaio: Avaliação e Políticas Públicas em Educação, Rio de Janeiro, v. 16, n. 60 , July/Sept. 2008.

GALVÃO, Zenaide. Educação física escolar: a prática do bom professor. Mackenzie de Educação Física e Esporte, São Paulo, v. 1, n. 1, p. 65-72. 2002.

GORGATTI, Márcia Greguol. Percepções dos docentes quanto à inclusão de estudantes com deficiência em aulas de educação física. Movimento, Porto Alegre, v. 15, n. 2, p. 119-140, abr./jun. 2009.

HAYCOCK, David; SMITH, Andy. To assist or not to assist? A study of teachers' views of the roles of learning support assistants in the provision of inclusive physical education in England. International Journal of Inclusive Education, Manchester, v. 15 , n. 8, p. 835-849, mar. 2011.

HAYCOCK, David; SMITH, Andy. Still 'more of the same for the more able?'Including young disabled people and pupils with special educational needs in extra-curricular physical education. Sport, Education and Society, London, v. 16, n. 4, p. 507-526, July 2011a.

HODGE, Samuel et al. High school general physical education teachers' behaviors and beliefs associated with inclusion. Sport, Education and Society, London, v. 9, n. 3, p. 395-419, 2004.

HOWES, Andrew J.; GRIMES, Peter; SHOHEL, M. Mahruf C. Imagining inclusive teachers: Contesting policy assumptions in relation to the development of inclusive practice in schools. Compare: a Journal of Comparative and International Education, Bath, v. 41, n. 5, p. 615-628, Apr. 2011.

HUTZLER, Yeshayahu. Attitudes toward the participation of individuals with disabilities in physical activity: A review. Quest, Atlanta, v. 55, n. 4, p. 347-373, Nov. 2003.

JEONG, Mihye; BLOCK, Martin. Physical education teachers' beliefs and intentions toward teaching students with disabilities. Research Quarterly for Exercise and Sport, Urbana-Champaign, v. 82, n. 2, p. 239-246, June 2011.

JERLINDER, Kajsa; DANERMARK, Berth; GILL, Peter. Swedish primary-school teachers' attitudes to inclusion: the case of PE and pupils with physical disabilities. European Journal of Special Needs Education, Coventry, v. 25, n. 1, p. 45-57. 2010.

KHOCHEN, Maha; RADFORD, Julie. Attitudes of teachers and headteachers towards inclusion in Lebanon. International Journal of Inclusive Education, Manchester, v. 16, n. 2, p. 139-153, 2012.

Movimento, Porto Alegre, v. 20, n. 2, p. 637-657, abr./jun. de 2014. 
KOWALSKI, Ellen; RIZZO, Terry. Factors influencing preservice student attitudes toward individuals with disabilities. Adapted Physical Activity Quarterly, Champaign, v. 13, n. 2 p. 180-196, April. 1996.

LAMBE, Jackie; BONES, Robert. Student teachers' perceptions about inclusive classroom teaching in Northern Ireland prior to teaching practice experience. European Journal of Special Needs Education, Coventry, v. 21, n. 2, p. 167-186, 2006.

MORLEY, David et al. Inclusive physical education: teachers' views of including pupils with special educational needs and/or disabilities in physical education. European Physical Education Review, Chester, v. 11, n. 1, p. 84-107, Feb. 2005.

NASCIMENTO, Karina Patrício et al. A formação do professor de Educação Física na atuação profissional inclusiva. Revista Mackenzie de Educação Física e Esporte, São Paulo, v. 6, n. 3. 2009.

OJOK, Patrick; WORMN/ES, Siri. Inclusion of pupils with intellectual disabilities: primary school teachers' attitudes and willingness in a rural area in Uganda. International Journal of Inclusive Education, Manchester, v. 1, n. 19, Oct. 2012.

PARRILLA, María Angeles; MORIÑA, Anabel. Criterios para la formación permanente del profesorado en el marco de la educación inclusiva. Revista de Educación, Sevilla, v. 339, 517-539, 2006.

PERRENOUD, Philippe. A pedagogia na escola das diferenças: fragmentos de uma sociologia do fracasso. Porto Alegre: Artmed, 2001.

PINTO PIRES, Filipa Isabel; CONDADO, Ricardo Jorge. Formação inicial de docentes de educação física face à inclusão de estudantes com necessidades. Wanceulen: Educación Física Digital, v. 9, p. 8-19, fev. 2012.

QI, Jing; HA, Amy Sau Ching. Hong Kong Physical Education Teachers' Beliefs about Teaching Students with Disabilities: a qualitative analysis. Asian Social Science, Leiden, v. 8, n. 8, p. 3, july. 2012.

RIZZO, Terry L.; DAVIS, Walter E.; TOUSSAINT, Ron. Inclusion in Regular Glasses: Breaking from Traditional Curricula. Journal of Physical Education, Recreation \& Dance, Reston, v. 65, n. 1, p. 24-47, 1994.

RIZZO, Terry; KIRKENDALL, Don. Teaching students with mild disabilities: What affects attitudes of future physical educators?. Adapted Physical Activity Quarterly, Champaign, v. 12, n. 3, p. 205-205, July. 1995.

RIZZO, Terry; VISPOEL, Walter. Physical educators' attributes and attitudes toward teaching students with handicaps. Adapted Physical Activity Quarterly, Champaign, v. 8, n. 1, p. 4-11, jan.1991.

RODRIGUES, David. Educação inclusiva: mais qualidade à diversidade. educação inclusiva e necessidades educacionais especiais. Santa Maria: Ed. UFSM. 2005.

Movimento, Porto Alegre, v. 20, n. 2, p. 637-657, abr.jun. de 2014. 
RODRIGUES, David. Inclusão e educação doze olhares sobre a educação inclusiva. São Paulo: Summus, 2006.

RODRIGUES, David. A Educação Física perante a Educação Inclusiva: reflexões conceptuais e metodológicas. Revista da Educação Física/UEM, Maringá, v. 14, n. 1, p. 67-73, 2008.

RODRIGUES, Heitor de Andrade; DARIDO, Suraya Cristina. The textbook in school Physical Education: a vision of teachers. Motriz: Revista de Educação Física, Rio Claro, v. 17, n. 1, p. 48-62, mar. 2011.

ROSS, Paulo Ricardo. Conhecimento e aprendizado cooperativo na inclusão. Educar em Revista, Curitiba, n. 23, n. 1. p. 203-224, 2004. Disponível em <http:// educa.fcc.org.br/scielo.php?script=sci_arttext\&pid=S0104-40602004000100012 \&lng=pt\&nrm=iso>. Acesso em: 3 jan. 2013.

SALVIA, John; MUNSON, Susan. Attitudes of regular education teachers toward mainstreaming mildly handicapped students. Mainstreaming Handicapped Children: Outcomes, Controversies, and New Directions. London: Lawrence Erlbaum, 1986.

SANCHES, Isabel; TEODORO, António. Da integração à inclusão escolar: cruzando perspectivas e conceitos. Revista Lusófona de Educação, Lisboa, v. 8 p. 63-83, jul. 2006.

SHARMA, Umesh; FORLIN, Chris; LOREMAN, Tim. Impact of training on pre $\square$ service teachers' attitudes and concerns about inclusive education and sentiments about persons with disabilities. Disability \& Society, Michigan, v. 23, n. 7, p. 773785, dez. 2008.

SOLER, Reinaldo. Brincando e aprendendo na Educação Física especial: planos de aula. 2. ed. Rio de Janeiro: Sprint, 2006.

SOUZA, Gracy Kelly Pereira; BOATO, Elvio Marcos. Inclusão de alunos com necessidades educacionais especiais nas aulas de educação física do ensino regular: concepções, atitudes e capacitação dos professores. Educação Física em Revista, Taguatinga, v. 3, n. 2, p. 1-15, 2010.

TRIPP, April; RIZZO, Terry L.; WEBBERT, Linda. Inclusion in physical education: Changing the culture. Journal of Physical Education, Recreation \& Dance, Reston, v. 78, n. 2, p. 32-48, Feb. 2007.

UNESCO, Organização das Nações Unidas para a Educação, Ciência e Cultura. Sobre princípios, políticas e práticas na área das necessidades educativas especiais. Salamanca/Espanha: UNESCO, 1994. 
Educação Física Inclusiva: Atitudes...

Endereço para correspondência:

Celina Luísa Raimundo Martins

Rua José Joaquim Ribeiro Teles, 566, habitação 4

4445-485 Ermesinde - Porto (PORTUGAL)

Recebido em: 24.05.2013

Aprovado em: 14.03.2014

Movimento, Porto Alegre, v. 20, n. 2, p. 637-657, abr.jun. de 2014. 